\title{
A New Step toward Mastering Double-Under Skill with Supporting Application of Image Processing
}

\author{
Kohta Sugawara*, Kenji Matsuura*, Stephen Karungaru* and Naka Gotoda**
}

(Received 24 December 2019 and accepted in revised form 4 August 2020)

\begin{abstract}
Rope skipping is a comparatively simple and easy exercise for people to perform solitarily anywhere. There are four stages for mastering double-under. The first two stages involve training the segmental motion, while the last two enhance the integration of the segmented motion mastered earlier. In the case of plyometrics like rope skipping, a learner may face tough challenges, requiring a fine adjustment of segmented motion at a faster pace and accurately in a short period of time. Therefore, this paper proposes a realignment of stage-organization with an additional step to address this challenge. We develop a supporting system so that learners can comprehend the essence and image of the target motion independently. The evaluation results indicate the effectiveness of our proposal.
\end{abstract}

Keywords: learning framework, learning support, integrated motor skill, rope skipping, visualized instruction

\section{Introduction}

People are in constant pursuit of acquiring new skills in various domains. The purpose is to engage in some activity like sports, music, dance and so on. In every case, there are some easy as well as arduous tasks. In the latter case, long term practice is generally necessary. During the process, a new technique or ability to execute the tasks is gradually accumulated. This paper deals with such techniques in exercise as a motor skill. It requires an advanced skill to execute the difficult tasks smoothly ${ }^{(1)}$. After acquiring primitive motor skills, people can attempt integrated motor skills that combine and coordinate the primitive motor skills.

There are some studies on creating an environment for acquiring motor skills. Gotoda et al. ${ }^{(2)}$ analyze a wide array of tricks in rope skipping and reveal their relationship on a skill map. This study supports the map tricks that have characteristics of both basic and advanced skills in rope skipping. An advanced skill combines and coordinates certain basic skills. When learners accomplish an advanced trick, they mainly treat two pieces of information as feedback. One is internal feedback, that is the performance results with trial and error, which is arrived at independently. The other is an external feedback, which is the performance evaluation observed by experts. Employing these responses, learners can modify their performance or recognize any mis-

\footnotetext{
*Tokushima University, Japan

**Kagawa University, Japan
}

representation regarding the goal. The latter is based on an expert's subjectivity, which is composed of some experiences and it tends to be tacit knowledge, because the experience is developed and stored as a model internally as a result of training for a long time. It suggests that once some parts of their performance are under consciousness with successful memory, the integrated skill is still under subconsciousness. Hence, the experience in the latest condition upgrades the didactic memory using the successful memory. Therefore, an instruction based on an expert who can perform special movements subconsciously after several attempts or trials may cause gaps in understanding during the learning process.

Gibson and Gibson ${ }^{(3)}$ describe perceptual learning as something a person can perceive that could not be perceived earlier. It is a way of thinking under which learning is to discover and focus on what could not be perceived previously. Specifically, it is to acquire a new variable $^{(4)}$. Therefore, it is essential that learners not only try to improve what experts recommend but also discover its essence and how to learn it independently ${ }^{(5)}$. Toward this end, it is imperative that a learner grasps the modification in both movement and overall performance in the case of an integrated motor skill. Hence, the present study applies asynchronous support for learners to confirm their own movement objectively ${ }^{(6)}$. The support is to provide learners with visible information:

(1) the quintessence that an expert executes

(2) difference between the learner and an expert on a 
video.

After observing them, the learner decides on a modified goal on a finer scale for the next training. Here, one needs to consider the differences among individuals because not all models of experts conform to a target learner since there are differences of performance even among experts. Hence, a goal set for a learner is determined by the analysis which calculates the performance parameter for a goal statistically in advance when experts' performances are digitized.

A learning process is crucial to achieve a goal. It is possible that learning many aspects for a short period of time or learning them simultaneously could result in amateurs not being able to understand or adapt to them. Consequently, their motivation will decline, or it could take a long time to accomplish the goal. The present study organizes several learning stages for successfully realizing a goal by controlling the number of learning points. There are four stages that are organized for stepby-step learning. The first two stages involve the training of segmental motion and the last two enhance the integration of the segmented motion mastered previously. However, while organizing these stages, some learners may face difficulties in mastering the third stage, because of the major step of integration from the second stage. Therefore, the study suggests a minor step that was originally introduced to bridge or reduce the large gaps between stages in preceding studies ${ }^{(7,8)}$.

\section{Learning Process}

\subsection{Motor Skill}

Skill can be defined in various contexts. Guthrie defines skill "as the ability to bring about some end result with maximum certainty and minimum outlay of energy or of time and energy"(9). Initially, maximum certainty is to adjust to the end result decided in advance, specifically, it depends on what degree a player can imitate target motion. Skill consists of several fundamental movements. The imitation is completed by understanding the structure and execution through trial and error. Accordingly, a player can express maximum certainty. Secondly, to execute with minimum energy implies saving their energy. The purpose is to make the best use of limited body resources, build muscle and range of motion. Consuming energy varies by the size of the muscle used during an exercise. Rope skipping requires a gross motor skill with greater movement. Finally, at the time of performing an exercise, rope skipping is classified as a plyometrics; one cannot stop execution in between because it is quick movement. Hence, in the case of plyometrics, a player prepares an exercise plan before execution.

\subsection{Integrated Motor Skill}

The present study defines integrated motor skill as an ability in which a player coordinates his/her body parts for controlling objects ${ }^{(10)}$. The related studies are as follows. Sasaki et al. ${ }^{(11)}$ provide learners with an environment to identify and address problems on their own. The environment consists of taking a video while performing target motion, processing it, and providing instructions to learners with the help of the processed video in order to acquire lay-up shot in basketball. This method can be applied to this study since a lay-up shot is an exercise of including an integrated motor skill. Kato et al. ${ }^{(12)}$ reveal the characteristics of an exercise by focusing on the relationship between body parts. One of the characteristics is that there is higher correlation between body parts for experts than amateurs on balllifting in soccer. This analysis method can be applied to rope skipping since ball-lifting is also an exercise that includes an integrated motor skill. The present study supports rope skipping as an exercise that includes an integrated motor skill adapting these methodologies.

\subsection{Rope Skipping}

Rope skipping is considered a life-long sport and a popular one because everyone from children to adults can perform it. One of the characteristics of the game is repetitive exercise which duplicates a segmented motion unit. This study considers the number of jumps or the execution time while observing the exercise. The other characteristic is to be able to regulate the intensity independently since there are both easy and advanced tricks. According to the skill map ${ }^{(2)}$, rope skipping tricks can be classified into two groups: single and double bounce. This indicates that the trick, single-under has a relationship with double-under as a fundamental trick in each group. Effectively, it is difficult for amateurs who can perform single-under to acquire double-under skills. One of the reasons is that amateurs have no idea how to move their body parts and how to coordinate them for 
double-under. The other reason is that it is difficult for them to adjust the rope timing. In double-under, the wrists should be rotated twice per jump. In addition, a player needs to control the speed because the cycle is different between the first and second rotation. Therefore, the present study focuses on a trick on performing double-under.

In this regard, the other related study is as follows. Yoshioka et al. ${ }^{(13)}$ analyze hand motion for doubleunder. In addition, the system of learning support provides beginners with visible information based on the image processing application. The information includes certain variations between a beginner's motion and the target motion. This study applies this learning environment to help beginners understand the difference. Yoshikawa et al. ${ }^{(14)}$ developed a system supported by sound worn by a learner for double-under. The purpose is that a learner estimates the difference of timing between body parts in order to remain stable. The research contends that learners remain stable in execution and move rhythmically by employing this system. Conversely, this study states that it is difficult for some players executing it in real time to recognize the timing of hand rotation accurately. In case of beginners in double-under, one needs to develop the timing. Therefore, the present study provides amateurs with a supporting system asynchronously.

\subsection{Method Proposal}

In this section, the study proposes learning methodology for double-under. Johnson ${ }^{(15)}$ classifies skill into four elements: speed, accuracy, form, and adaptability. It is desirable for beginners who learn motor skills to acquire form, accuracy, speed, and adaptability in that order. Two related studies by Yoshioka et al. ${ }^{(13)}$ and Sugawara et al. ${ }^{(8)}$ are corresponded with acquiring the form in double-under. Therefore, this study considers the accuracy and speed in addition to acquiring the form.

Ohta $^{(16)}$ discusses strategies to promote motor skills based on the dynamics of the human body or an operating object. It is a way of conveying a skill by a potential format or an appealing one, not a manifest one, such as verbalization or visualization of a motor skill. Doubleunder has an aspect of plyometrics. If a player cannot execute it, the exercise is terminated. Meanwhile, it has another aspect of a repetitive exercise, so that a learner can train without failing by repeating a series of motion units decided in advance. The present study proposes a training schedule considering the dynamics and both sides in double-under. Under this, a learner trains with an arranged rope that is cut into two and with an appropriate weight attached at the top of two ropes. A learner can execute double-under artificially with the rope. The present study defines this training as a small-bridging step in double-under.

ARSA, which stands for Australian Rope Skipping Association, introduces learning stages for a trick of rope skipping. ARSA does not act extrinsically with its original name, but its document is still open and available for access ${ }^{1}$. Figure 1, which illustrates the structure of learning stages, inserted a middle stage (3') based on the learning model of ARSA. Stage 1 and 2 are fundamental stages where a learner practices certain primitive movements independently in each stage without a rope. Stage 3, 3', and 4 are advanced stages where a learner practices to coordinate primitive movements by combining hand and jump motion. A learner practices without a rope in Stage 3, with an arranged rope in Stage 3' and with a rope in Stage 4.

In the proposed Stage 3', learners do not mind getting entangled in the rope and feel free to try the integration of multiple body movements simultaneously. This stage has a criterion to estimate whether or not the goal is achieved. The performance is judged by monitoring the movement of the top of the rope. If it passes

\begin{tabular}{|lc|}
\hline Stage 1 & $\begin{array}{c}\text { Hand m otion } \\
\text { without a rope }\end{array}$ \\
\hline Stage 2 & $\begin{array}{c}\text { Jum p m otion } \\
\text { without a rope }\end{array}$ \\
\hline $\begin{array}{r}\text { Stage 3 } \\
\text { Hand \& Jum p m otion } \\
\text { without a rope }\end{array}$ \\
\hline Stage 3' 4 & $\begin{array}{r}\text { Hand \& Jum p m otion } \\
\text { with an arranged rope }\end{array}$ \\
\hline
\end{tabular}

Figure 1. Arranged Learning Stages for Double-under.

\footnotetext{
${ }^{1}$ Double-under stages described in the manual https://www.sports-media.be/links/Coaching-Manual-RopeSkipping.pdf (last accessed 2020.6.5)
} 
through the foot at the time of landing, the system sets it as successful, otherwise it is recorded as a failure (see Figure 2).

\section{System Environment}

\subsection{Overview of the System}

$\mathrm{VC}++$.Net is used to develop a prototype system which incorporates OpenCV library for image processing technology applying on the captured video. The configuration of the system is illustrated in Figure 3. There are three major modules in this system: (a) feature point detection, (2) analysis and estimation associated with the database, and (3) output generation.

\subsection{Supporting Functions}

In the concrete sequence of system usage, a beginner uploads a captured video file to the system through

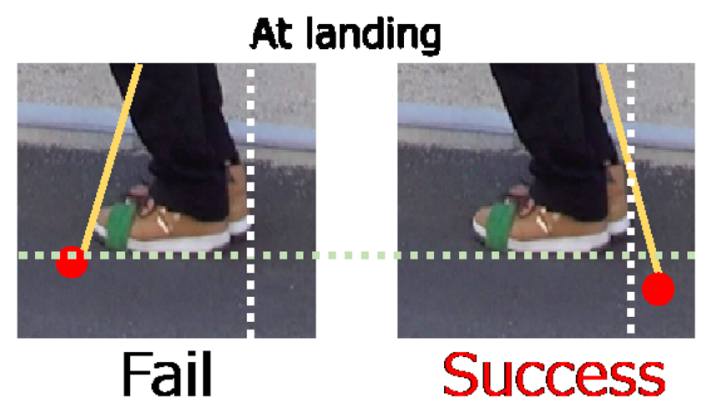

Figure 2. Success/Failure at Landing.

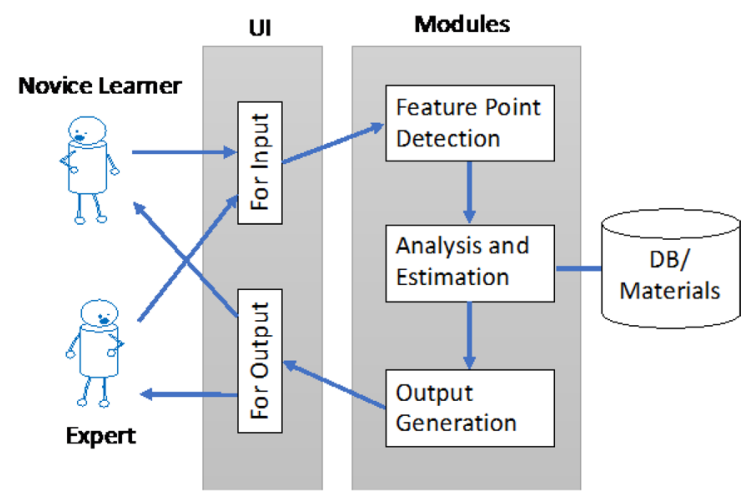

Figure 3. System Configuration. the user interface, whose acronym is "UI" in Figure 3. $\mathrm{S} /$ he prepares his/her movie file made previously by a video-camera which monitored his/her rope-skipping. Capturing the motion into the video file is performed by a camera set at the side of the body. One side camera does not matter because the target trick is double-under, where it is unnecessary for cross motion of arms, at this time. When the learner completes uploading the operation, the movie file is processed by an image processing application and some features are automatically detected by using color-markers. In terms of component technique regarding feature points detection, Karungaru et al. proposed the integration of particle filter for estimating rope skipping motions ${ }^{(17)}$. Through the process, we can verify the motion trajectory of the hands and arms, for instance.

\subsection{Analysis and Estimation}

Rope-skipping is composed of roughly two major movements, which are skipping in the vertical direction and swinging motion of arms in an orbicular way.

Since double-under movement does not need crossover action of the arms, we can monitor the arm-swing in approximately two dimensions. In addition, both arms synchronize mutually in many cases, only one-side recording is enough to capture the movements as already mentioned. Tracing the two-dimensional movement of the arm is performed with smoothing technique in image-processing by an original application using OpenCV library. Smoothing includes linear-approximation, moving-average and Region of Interest (ROI) functionality. Therefore, the system identifies four parts of local maximum point of the acceleration as shown in Figure 4. Since there is a variety of styles depending on the learner, we have asked eight experts to play doubleunder and collected 188 sets of data thereof in total. Furthermore, the detection of four local maximum points of acceleration is not always performed, yet there were approximately four segments of time measured by frame counts in a skip. Since a cycle of a skip is different at each skip, we have converted the four frame lengths into ratio. As a result, mean values of such percentage are $22.26,24.00,24.01$, and 29.55 , respectively. Using the segmentation in such a way, we can detect the local maximum within each segmentation in a relatively easy manner. The higher acceleration indicates the timing of empowering to gain in velocity to the rope. In this 

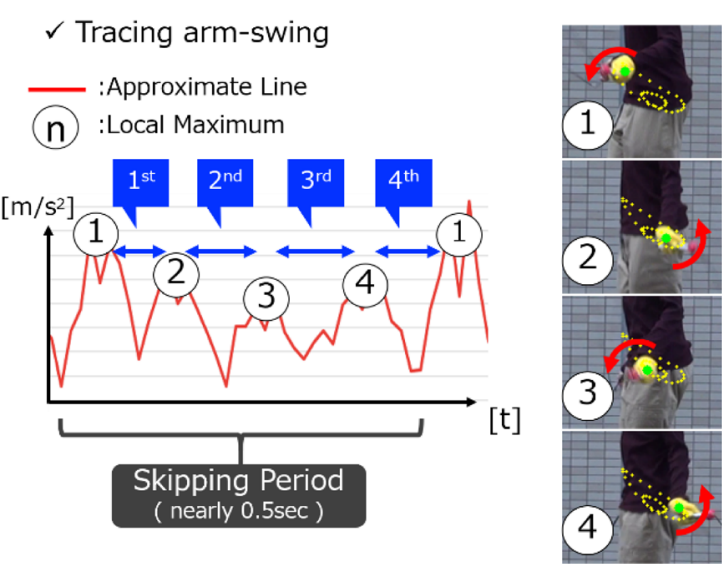

Figure 4. Tracing Movement Trajectory of Arm-swing and its Approximate Line.

Table 1. C.I. of Criteria by Experts.

\begin{tabular}{|l|c|c|}
\hline \multicolumn{1}{|c|}{ Item } & Lowest of C.I. & Highest of C.I. \\
\hline $\begin{array}{l}\text { H1 } \\
\text { (Radius of arm } \\
\text { swing trajectory) }\end{array}$ & $0.05593[\mathrm{~m}]$ & 0.07932 [m] \\
\hline $\begin{array}{l}\text { H2 } \\
\text { (Period of wrist- } \\
\text { movement cycle) }\end{array}$ & 16.25 [frames] & 17.88 [frames] \\
\hline $\begin{array}{l}\text { J1 } \\
\text { (Duration in the } \\
\text { jumping) }\end{array}$ & 27.97 [frames] & 31.38 [frames] \\
\hline $\begin{array}{l}\text { J2 } \\
\text { (Relative size of the } \\
\text { body in landing) }\end{array}$ & 90.75 [\%] & $92.45[\%]$ \\
\hline $\begin{array}{l}\text { J3 } \\
\text { (Relative size of the } \\
\text { body in the air) }\end{array}$ & 81.69 [\%] & $89.52[\%]$ \\
\hline
\end{tabular}

manner, the radius, the speed and the rhythm of the circular orbit of arm-swing can be measured.

Tracking the jump is much simpler. The player wears color markers on the tips of the toes, for instance. As image processing technique can be applied here to detect the marker easily and the result is converted to the wave form in a time line. Detecting height and posture of the foot in both landing and jumping are available to use for estimation. Therefore, overall, six items including rhythm in addition to items in Table 1 are adopted.

The indicator $\mathrm{H} 1$ demonstrates the estimated radius which is the mean value of the distance from centroid to the tracking point. Both $\mathrm{H} 2$ and $\mathrm{J} 1$ are measured as length of frames. Both $\mathrm{J} 2$ and $\mathrm{J} 3$ are ratios which is the relative size against the natural size under a normal standing condition. When a learner is 170 centimeters tall, the pixel size from the head to toe should be $100 \%$. When s/he jump and the length from the top of the body to the bottom is $80[\%]$ in pixel length, the system identifies the relative length of the body to 0.8 , specifically $80[\%]$.

Using these items, the system can identify whether the learner has succeeded or not. Especially in Stage 3', Figure 2 of Section 2.4 is applied in an implementation.

In order to prepare the acceptable range of referential values of experts, we obtained data of ten trials by experts. The number of experts were six who tried double-under and succeeded ten times simultaneously. Using the data obtained, we calculated the confidence interval, C.I., of $95[\%]$ in a statistical manner (see Table 1). As for the rhythm, Fourier transformation is used to check if two peaks of frequencies appeared. In the experiment, we use these values for judging success or failure of the trials by the learners. The system counts up the successful number of jumps from these perspectives and calculating the rate against the total number of jumps is used for actual judging. For instance, when six times is counted as successful, that is within the C.I. values against a total ten jumps, the estimation is performed with 0.6. The values in $\mathrm{H} 2$ and $\mathrm{J} 1$ are the detected number of frames whose frame rate is 60 [fps]. Judging from the monitored values shown in the table, $500[\mathrm{msec}]$ is the approximate average period of one jumping movement.

Correlation coefficient is calculated independently among values in any combination of items. The highest correlation coefficient is recorded in the combination of $\mathrm{H} 2$ and $\mathrm{J} 1$, whose score is 0.8812 . Therefore, in the experiment, we mainly use either of the items, specifically $\mathrm{H} 2$, as a period indicator.

\subsection{System Interface}

An effective prototype system presents a user interface only in Japanese, a snapshot of which is seen in Figure 5. The figure illustrates six area-blocks as follows; 


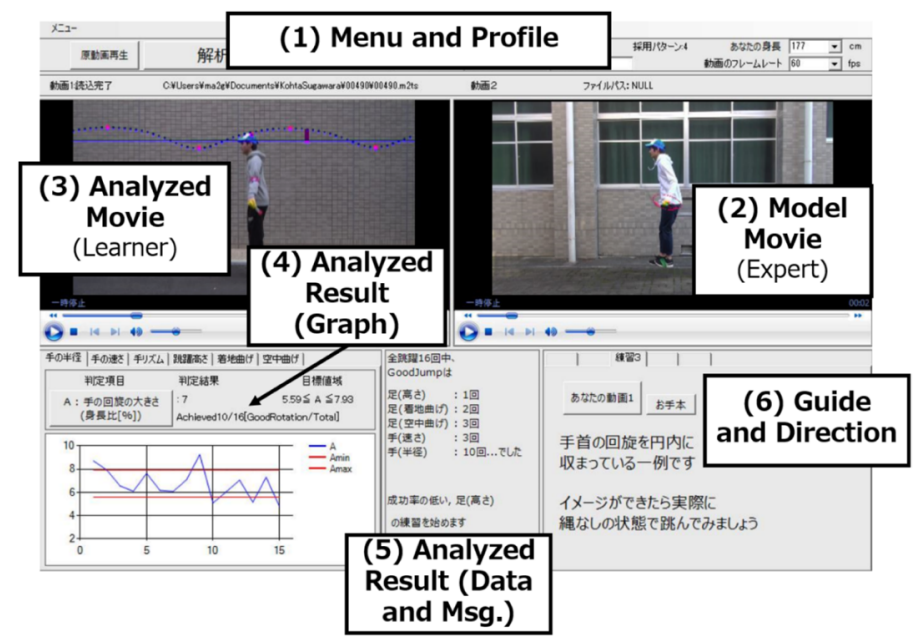

Figure 5. A Snapshot of the System.

(1) Main menu

It is used for selecting the content and profile data of a learner. The upper area provides the menu with some profile information input by a learner. Here, for example, placing a button prompts a system to start the analysis. It is also possible to adjust the replaying speed of a video that is shown below the menu.

(2) Model movie of a referential expert

The system needs model movies to show a learner. Therefore, we collected movies of experts successively for reference and stored their information associated with the video materials into the system in advance. A learner can select one of them after referring to the information or reviewing the material. Subsequently, a selected item appears assigned at the middle right. There are individual specificities in both physicality and the way of skipping. Therefore, a learner must select an appropriate one for the reference.

A system provides three kinds of analyzed information of a learner as follows:

(3) Analyzed Movie of a learner

(4) Analyzed Result (Graph area)

(5) Analyzed Result (Data and messages)

The analysis of a movie, which is the input by a learner, is carried out by revealing the path of a movie to the system. When completing the processing, a frame on the area (3) begins its processed movie with remarkable symbols superimposed. In parallel, several types of analysis provide graphic representation in area (4) with associated numeric data in area (5) whose concrete method of analysis is described in the next section.

The actual data shown as (4) in Figure 4 is the radius represented in a line graph style which connects the measured radius value at each jump. The system determines whether or not it is a success by verifying whether the values ranged from $5.59[\mathrm{~cm}]$ to $7.93[\mathrm{~cm}]$ calculated in C.I. (see Section 3.3). A total of 10 points are considered to be within the accepted range in 16 jumps overall.

The data shown in (5) is the counts of success in five categories of indicators, which include the radius of the arm-swing trajectory, period of wrist movement cycle, duration in the jumping, relative size of the body in landing, relative size of the body in the air. The message is "Let's practice focusing on \#\#\#\#\# which is a relatively low rate of success." where \#\#\#\#\# is replaced with one of the five categories described above.

(6) Guide and direction messages

A set of detected information are associated with a message within a fixed list of types. The selection indicates that the system assesses the current learner and therefore the next challenge of training for a learner is also decided. As a result, the message shown here in area (6) involves the key points for the current trial and direction for the next. The messages shown here are previously defined with the stored model video. The concrete mes- 
Table 2. Messages associated with the items in Table 1.

\begin{tabular}{|l|l|}
\hline Item in Table 1 & $\begin{array}{l}\text { Messages translated into English from } \\
\text { original Japanese (Case stage 3). }\end{array}$ \\
\hline H1 & $\begin{array}{l}\text { Refer to the model video, which is a good } \\
\text { example regarding the radius of arm-swing } \\
\text { trajectory. When you get the clear picture, } \\
\text { practice without a rope. }\end{array}$ \\
\hline H2 & $\begin{array}{l}\text { Learn the appropriate timing by way of } \\
\text { rotating your wrist quickly at the time you } \\
\text { identify a luminous shape of estimated } \\
\text { human pose. When you get the clear pic- } \\
\text { ture, practice without a rope. }\end{array}$ \\
\hline J1 & $\begin{array}{l}\text { Become strongly conscious of height in } \\
\text { jumping. When you get the clear picture, } \\
\text { practice without a rope. }\end{array}$ \\
\hline J2 & $\begin{array}{l}\text { Refer to the model video, which is a good } \\
\text { example regarding the relative size of the } \\
\text { body in the air. When you get the clear pic- } \\
\text { ture, practice without a rope. }\end{array}$ \\
\hline J3 & $\begin{array}{l}\text { Refer to the model video, which is a good } \\
\text { example regarding the relative size of the } \\
\text { body at landing. When you get the clear } \\
\text { picture, practice without a rope. }\end{array}$ \\
\hline
\end{tabular}

sages are originally in Japanese but the translation into English is listed in Table 2.

\section{Trial Use and Evaluation}

\subsection{Organization and Procedure of the Experiment}

We conducted an experiment with 14 volunteer subjects in their 20s who had already mastered skills in Stages 1 and 2. Therefore, they did not require these two stages in terms of experimental tasks assigned. They wore markers on the top of the head, wrist, and instep with different colors. They also used two types of rope; normal one at Stage 4 and a pair of special-cut ones for Stage 3'. The duration of the experiment was three weeks and each subject could try with enough space where the camera recording had no stress with any ambiguous background. The number of jumps in one trial was eight on average, but we picked up five consecutive sets, excluding the first and the last jump in order to avoid noise which were a different movement in a jumping style. Despite the number of jumps, the subjects neither complained nor had difficulties of physical fitness.

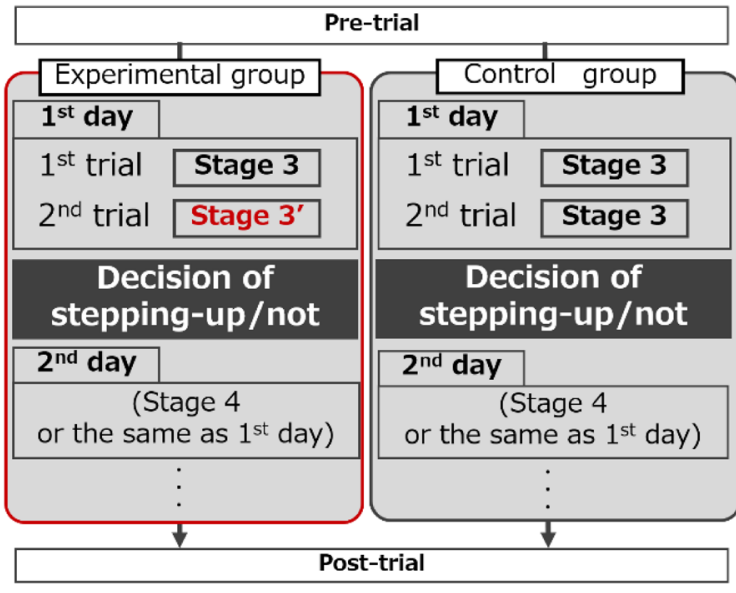

Figure 6. Organization of the Experiment.

The overall structure of the experiment is shown in Figure 6 . All the subjects were asked to perform the trials at beginning and answer a questionnaire regarding their experiences about rope-skipping at that time. They were also asked to perform post-trial for verifying the effects without a system support. They trained themselves between these processes and were asked to answer the questionnaire for verifying the explicit recognition for success at each instance. The experimental group used a proposed system with Stage $3^{\prime}$ and the control group used the normal system without Stage 3'. We evaluated the performance each day in order to decide whether the target subject could proceed to the next advanced stage or not. Six training days were distributed to every subject within three weeks.

A pre-phase questionnaire asked experiences of rope-skipping, and pre-trial was used for forming two homogeneous groups in the experimental (named group A) and control (named group B) ones. Pre-trial included single-bounce with a rope, double-bounce without a rope and with a rope. The difference between the groups was only at Stage 3', which pertained to information such as text-based summary and suggestions of the next-time training in addition to the different graph representation.

In the decision, we set an average score of succeeding items, which is within the values of C.I. described in Table 1. using eq. (1), where $i$ is the label in six items, $j$ is the index of trials, $n$ is the total number of jumps, and $O_{i}(k)$ is 1 if the item $i$ at $k$ is within the C.I., otherwise it 
sets 0 .

$$
Q_{i, j}=\frac{1}{n} \sum_{k=1}^{n} O_{i}(k)
$$

The value $Q_{i, j}$ can be regarded as the indicator of the performance score with respect to the item $i$ at trial $j$. We successfully made homogeneous groups using this value from a statistical viewpoint. The actual mean value and standard deviation of success in each group were $25.55(N=7$, s.d. 10.32) and $25.39(N=7$, s.d. 10.78), respectively, at the pre-trial phase.

\subsection{Evaluation of Obtained Data as the Result}

(1) Successful attempts

Initially, continuous success was registered in both the groups. At the pre-trial phase, the number of players achieving double-under more than once was three in both groups, totaling seven members. The number of successful players was similar in both the groups and the time of continuing was one by each player. After the experiment at post-trial, the number of achievements was seven in group A and six in group B. However, the distribution of continuing times varied. We tested the continuing times of each group using the Brunner-Munzel's test, which did not assume normal distribution nor homoscedasticity. As a result, we obtained a p-value of 0.057 $(<.10)$, which indicates a significant difference of $10 \%$.

(2) Effect of a new step

Since the system offered Stage 3' for only group A, we verified the difference of performance scores at the pre-phase and post-phase stage in that group. We obtained a p-value of $0.026(<.05)$ by employing the Welch's test, which indicated a significance level of $5 \%$.

(3) Comparison of each item

The system presents feedback information on each item as described in Table 1. Here we checked the performance scores of each item by comparing them within the two groups. There we found two interesting results in $\mathrm{J} 2$ and $\mathrm{H} 2 . \mathrm{J} 2$ is the performance score of the relative body size in landing where normal height at stable posture is set as 1.00 . When the improvement from prephase to post-phase was compared as the effect of the training, we found a p-value of $0.060(<.10)$ through the Welch's test. Next is H2, which is a score for the period of the wrist movement cycle. The wrist movement needs relatively more training than others because it is a kind of plyometric exercise. Based on this belief, we verified the improvement score by the system support. It implies that the comparison is made for the main training phase. Thereafter, we obtained the difference in performance score significantly, p-value $0.013(<.05)$ by the Welch's test.

(4) Questionnaire

The results of the questionnaires asking the recognition at the training phase were examined. Both the scores before the training and after the training were almost the same from a statistical perspective. The scores of recognitions for performance improvement were not significantly different, although some actual performance scores indicated significance.

\subsection{Discussion}

With respect to the difference of recognition, the results of questionnaire (4) of the former section indicates almost the same score between groups. It suggests that they intended to play rope-skipping in a better way from their own perspectives. However, there were significant differences in the two criteria described in (3) of Section 4.2. At the outset, the posture at landing was improved to match with the experts. It is a relatively easy challenge for learners by performing consciously. Therefore, we should recommend designing the posture based on the expert's position. Next, we found improvements in $\mathrm{H} 2$ during the training supported by the system. As the speed of the wrist movement was higher than other body parts, system support was very useful for immediate reflection with improvement.

According to (1) in Section 4.2, overall improvement was recorded in the experimental group, which was identified successively in times of consecutive double-under. Six members could successfully play doubleunder with more than one jump in the experimental group, compared to only in a control group.

We received subjective comments from learners in this experiment. A learner from group B claimed that he had experienced difficulty in rope handling rather than the expectation from the rope-less training phase, Stage 3 . This was actually one of our motivations to reduce the gap between such stages gradually. We also received another comment from a subject in group B. He believed that he performed involuntary movement with different styles between stages with a rope and without a 


\section{A NEW STEP TOWARD MASTERING DOUBLE-UNDER SKILL WITH SUPPORTING APPLICATION OF IMAGE PROCESSING}

rope. This interrelates another motivation of our proposal for improvement.

\section{Conclusions}

The study proposed incorporating a small new step for the double-under training of the ARSA model. When a beginner experiences difficulty in approaching one training stage from another, a kind of scaffolding between the two stages with a high gap contributes to the interpolation. In the actual implementation of the new stage inserted, we designed and implemented the technical solution for analysis and feedback. Our project evaluated the prototype focusing on the double-under in this context and received several findings described in Section 4.3.

As for future implications, we should apply this method to other tricks in rope skipping or other similar movements.

\section{Acknowledgement}

This work was supported by JSPS KAKENHI Grant Numbers JP18H03344, JP15K01072. In terms of system implementation, an alumni, Mr. Shinya Yoshioka contributed significantly on this work.

\section{References}

(1) Furukawa, K. et al: Introduction to Skill Science, Ohmsha, Japan (2009).

(2) Gotoda, N., Matsuura, K., Nabeshima, T. et al.: "Supporting Both Blog-Article Accessing and Skill Overviewing on Nawatobi-SNS", Japan Society for Educational Technology, Vol. 34, No. 3, pp. 269-277 (2010).

(3) Gibson, J. J. and Gibson, E. J.: "Perceptual Learning: Differentiation or Enrichment?", Psychological Review, Vol. 62, No. 1, pp. 32-41 (1955).

(4) Furukawa, K., Suwa, M. and Kato, T.: "On Supporting Physical Skill Discovery", Transactions of the Japanese Society for Artificial Intelligence, Vol. 22, No. 5, pp. 563573 (2007).

(5) Suwa, M.: "Constructive Perception: Coordinating Perception and Conception toward Acts of Problemfinding in a Creative Experience", Japanese Psychological Research, Vol. 45, No. 4, pp. 221-234 (2003).

(6) Murayama, M., Muramatsu, T., Sasaki, R. et al: "Effect of Immediate Image Feedback of a Motion Image Analysis in
Skills Training during Early Stage Teaching of a Sidearm Throw in Flying Disk Sports", Bulletin of the institute of physical education, Keio university, Vol. 46, No. 1, pp. 1-15 (2007).

(7) Cho, S., Hofuku, Y., Nishida, T. et al.: "De-gapper: Tool for Support Programming Learners" "Step-by-step" Learning", Information Processing Society of Japan, Vol. 55, No. 1, pp. 45-56 (2014).

(8) Sugawara, K., Toyooka, H., Matsuura, K. et al.: "Development of a Gross Motor Skill by Combining Primitive Motions in Repetitive Exercise", 2016 IEEE $5^{\text {th }}$ Global Conference on Consumer Electronics, pp. 488-492 (2016).

(9) Guthrie, E. R.: “The Psychology of Learning”, Harper's Psychological Series, Harper (1952).

(10) Furukawa, K., Ueno, K., Ozaki, T. et al.: "Research Trend of Physical Skill Science: Towards Elucidation of Physical Skill”, Transactions of the Japanese Society for Artificial Intelligence, Vol. 20, No. 2, pp. 117-128 (2005).

(11) Sasaki, N.: "Effects of Visual Feedback for Skill Acquisition", Bulletin of Biwako Seikei Sport College, Vol. 8, pp. 121-127 (2011).

(12) Kato, K., Namiki, Y., Thepvilojanapong, N. et al.: "Exploring Analysis of Football Actions Considering Correlation of Joints in the Body", in 2012 Ninth International Conference on Networked Sensing (INSS), pp. 1-2 (2012).

(13) Yoshioka, S., Matsuura, K. and Gotoda, N.: "Hand-motion Analysis for Development of Double-unders Skill", Procedia Computer Science, Vol. 60, pp. 775-783 (2015).

(14) Yoshikawa, T., Matsuura, K., Sugawara, K. et al.: "Supporting Stabilization of Timing Difference between Coordinated Motor Actions in Double-under by Sensing Multiple Body Parts", Japan Society for Educational Technology, Vol. 41, No. Suppl., pp. 229-232 (2017) (in Japanese).

(15) Johnson, H. W.: "Skill $=$ speed $\times$ accuracy $\times$ form $\times$ adaptabili ty", Perceptual and Motor Skills, Vol. 13, No. 2, pp. 163170 (1961)

(16) Ohta, K.: "A Cybernetic Training Supporting for Acquiring Motor Skill", J. of the Society of Biomechanisms Japan, Vol. 37, No. 4, pp. 227-232 (2013) (in Japanese).

(17) Karungaru, S., Matsuura, K. and Gotoda, N.: "Feature Tracking Using Particle Filter in Rope Skipping for Gross Motor Skill Development," IJCA Proc. on International Conference on Cognitive Knowledge Engineering, Vol. ICKE 2016, No. 1, pp. 25-29 (2018). 


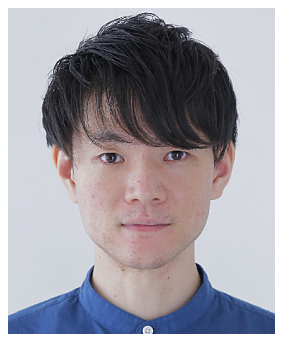

Kohta Sugawara received the B.E. and M.E. from Tokushima University in 2016 and 2018 respectively. He is currently working with Fujitsu Limited. His research interests include information systems and learning science.

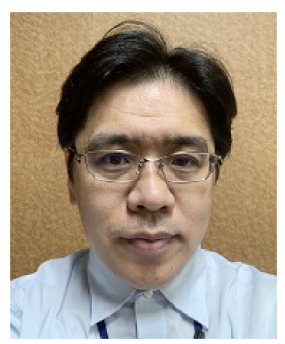

Kenji Matsuura received the B.E. and M.E. from Tokushima University in 1994 and 1996 respectively. He received the Ph.D. from Tokushima University in 2002 . He is currently a professor, Tokushima University. His research interests include human-skill development. $\mathrm{He}$ is a member of JSiSE, JSET, IPSJ, JSAI and IEICE.

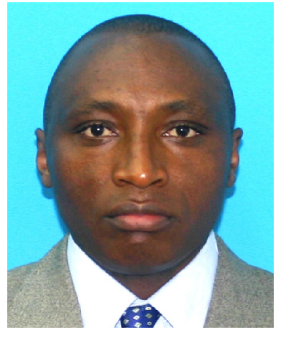

Stephen Karungaru received a Master's and a doctoral degree from the Department of Information Science and Intelligent systems, Faculty of Engineering, Tokushima University, in 2001 and 2004 respectively. He became an Associate Professor, Institute of Advanced Science and Technology, Tokushima University since 2010 . His research interests are in Face Detection, Recognition, Neural Networks, and Genetic Algorithms.

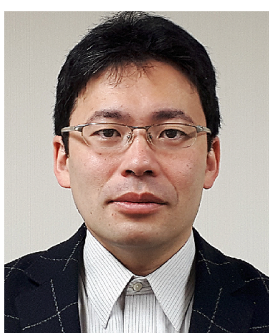

Naka Gotoda received the M.E.and $\mathrm{Ph} . \mathrm{D}$. from Tokushima University in 2008 and 2010 respectively. $\mathrm{He}$ is currently an associate professor of Kagawa University. His research interests include skill science. He is a member of IEICE, HIS, JSiSE, JSAI and AIJ. 